
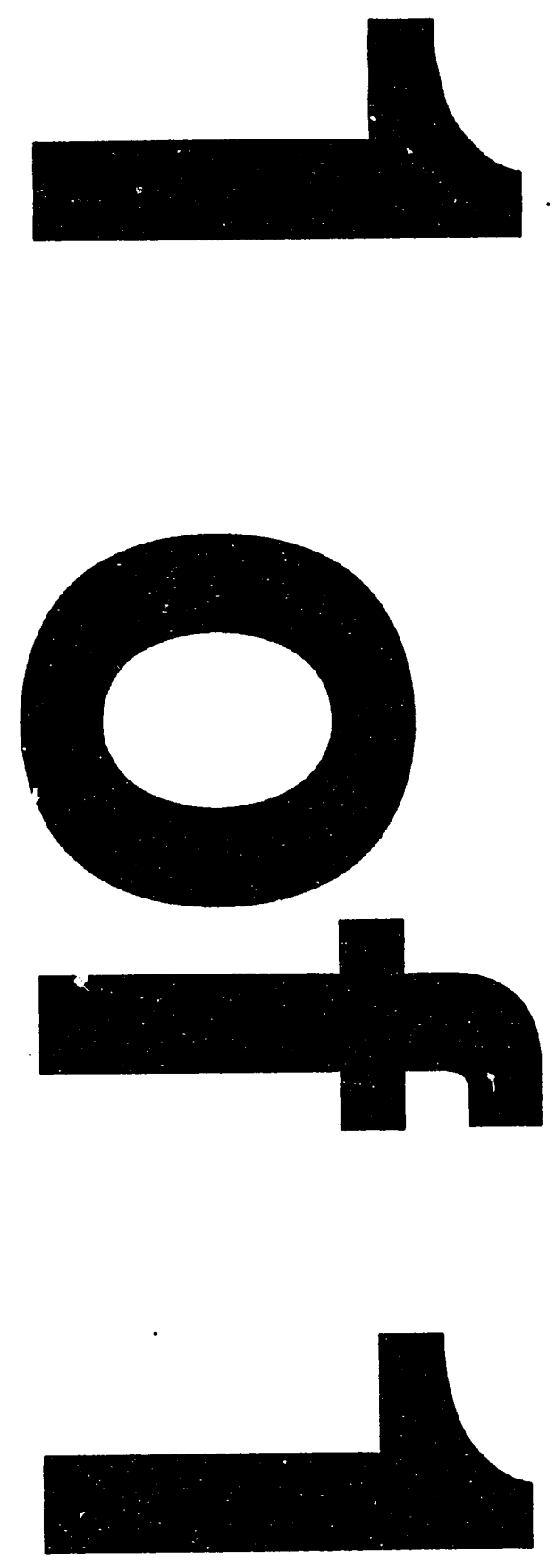


\title{
TOWARD A SOFT X-RAY FOURIER-TRANSFORM SPECTROMETER
}

\author{
M.R. Howells, K. Frank, Z. Hussain, E.J. Moler, T. Reich, D. Möller, D.A. Shirley
}

\author{
Advanced Light Source \\ Accelerator and Fusion Research Division \\ Lawrence Berkeley Laboratory \\ University of California \\ Berkeley,CA 94720
}

October 29, 1993

Paper Presented at the 8th National Conference on Synchrotron Radiation Instrumentation, Gaithersburg, Maryland, USA, August 23, 1993-August 26, 1993.

"This work was supported by the Director, Office of Energy Research, Office of Basic Energy Sciences, Materials Sciences Division of the U.S. Department of Energy, under Contract no. DE-AC03-76SF00098 


\title{
TOWARD A SOFT X-RAY FOURIER-TRANSFORM SPECTROMETER
}

\author{
M. R. Howells ${ }^{1}$, K. Frank ${ }^{1}$, Z. Hussain'1, E. J. Moler ${ }^{1}$, T. Reich ${ }^{1,3}$, D. Möller², D. A. Shirley³ \\ 'Lawrence Berkeley Laboratory, Berkeley CA 94720 USA. \\ 2Department of Physics and Chemistry, Fairleigh Dickenson University, Teaneck NJ 07666 USA. \\ ${ }^{3}$ Departments of Chemistry and Physics, Pennsylvania State University, University Park, PA 16802
} USA.

\section{ABSTRACT}

The use of Fourier transform spectroscopy (FTS) in the soft x-ray region is advocated as a possible route to spectral resolution superior to that attainable with a grating system. A technical plan is described for applying FTS to the study of the absorption spectrum of helium in the region of double ionization around 60-80 eV. The proposed scheme includes a Mach-Zehnder interferometer deformed into a rhombus shape to provide grazing incidence reflections. The path difference between the interfering beams is to be tuned by translation of a table carrying four mirrors over a range $\pm 1 \mathrm{~cm}$ which, in the absence of errors generating relative tilts of the wave fronts, would provide a resolving power equal to the number of waves of path difference: half a million at $65 \mathrm{eV}$, for example. The signal-tonoise ratio of the spectrum is analyzed and for operation on an Advanced Light Source bending magnet beam line should be about 330 .

\section{INTRODUCTION}

The resolving power of any spectroscopic instrument is essentially equal to the number of waves of path difference between the rays of greatest and least path through the instrument. In synchrotron radiation practice one mainly encounters systems based on gratings and crystals operated in low order, usually first, but these are not the only possibilities. Spectrometers based on both prisms and FabryPerot etalons are widely used in the visible region and are also governed by the above principle [1]. However, such spectrometers are not normally used outside the transmission region of glass and consequently are not all that useful for synchrotron spectroscopy. Our interest in this paper is in another class of spectrometers which utilize a two-beam interferometer (usually a Michelson type) to measure the Fourier transform of the desired spectrum. These devices have been widely used in the infrared (including that derived from synchrotrons [2]) and the near UV [3] and we now propose that the same concept be adapted for use in the soft $x$-ray region. This will enable the principle of Fourier transform spectroscopy (FTS) to be applied to soft x-ray photoabsorption experiments with the prospect of obtaining higher resolution than is possible with a grating. The path difference between the interfering beams in FTS is provided by means of a moving mirror and the resolving power is therefore determined by the amount of path difference allowed by the mirror mechanical system. This is a quite different type of limitation than the ones normally encountered in grating systems which is why we believe that FTS may provide a route to higher resolution. It is aiready demonstrated that, given an interferometer with the required path difference, FTS can achieve a resolving power greater than a million in the near UV [3]. Moreover, as we explain in the next section there are a number of scientific applications which would benefit from a resolving power of this order. The one of particular interest to us is the study of helium by absorption spectroscopy in the energy range $60-80 \mathrm{eV}$ near the double-ionization threshold.

The use of FTS in the soft $x$-ray region has never been attempted as far as we know although it has been discussed [4]. We believe that a two-beam interferometer operating down to $10 \mathrm{~nm}$ is technically within reach and we outline our proposal for it in this paper. Our scheme is essentially an 
adjustable-path Mach-Zehnder interferometer [5] with the usual square layout deformed to a rhombus to give grazing reflections of suitable angle. The target wavelength range is $10-100 \mathrm{~nm}$ and the maximum path difference is set at one millin 7 waves at $10 \mathrm{~nm}$ which is one centimeter.

In the sections that follow we discuss some of the general issues of interferometric spectroscopy and contrast the performance of interferometers and gratings with regard to resolution, phase space acceptance and noise. We describe our technical plan in detail with particular emphasis on the two most challenging aspects which we consider to be the beam splitters and the mechanical motion system. We conclude by providing an assessment of the performance we expect from our proposed interferometer when it is fed by a monochromator on an Advanced Light Source (ALS) bending magnet beam line.

\section{SCIENTIFIC MOTIVATION}

Our group has a particular interest in photoabsorption studies of helium. Such studies have attracted considerable interest both from experimentalists and theoreticians during the last three decades for several reasons. Helium is the simplest atom for which the Schrödinger equation cannot be solved analytically. With only two electrons, it is a prototype three-body system in atomic physics and is important for the study of electron-electron correlations. Of special interest is the spectral region from 60 to $80 \mathrm{eV}$, where both electrons are excited. The pioneering work by Madden and Codling in 1963 revealed two series of autoionizing states below the $\mathrm{N}=2$ threshold of $\mathrm{He}^{+}[6]$. Soon after this experiment, the shape and very small width of these resonances were explained by the relatively weak coupling of the autoionizing states to the continuum [7, 8]. Further theoretical understanding led to the formulation of several sets of approximate quantum numbers [9-12]. It took nearly three decades before, in 1990, a soft $x$-ray monochromator with a resolving power of $E / \Delta E=10,000$ allowed the observation more than 50 states below the $\mathrm{N}=2-7$ threshold of $\mathrm{He}^{+}$some of which were as narrow as $0.1 \mathrm{meV}$ (see Fig. 1) [13]. This experiment challenges the theory to calculate adequate values for the position, shape, and intensity of these Rydberg lines, which for higher $\mathrm{N}$ are increasingly complicated by interchannel interferences. Further improvement of the resolution from 6 to $4 \mathrm{meV}$ allowed the experimental detection of the lowest four members of the theoretically predicted $2(-1,0)_{n}^{0}$ Rydberg series for the first time [14]. These resonances are a sensitive test of the theory, which in some cases has failed to predict the right sequence of the $2(1,0)_{n}^{-}$and $2(-1,0)_{n}^{0}$ series. Even the latest $4 \mathrm{meV}$ resolution is not sufficient to determine any of the line-shape parameters for these extremely narrow resonances. Such values are crucial for the test of theories, which disagree by two orders of magnitude with respect to the width of the $2(-1,0)_{3}^{0}$ state, for example, the calculated values ranging from $4 \times 10^{-5}$ to $3 \times 10^{-3} \mathrm{meV}[15,16]$. Such experimental evidence as there is presently [14] agrees with neither of these values. As revealing as the latest high-resolution results have been they clearly demonstrate the need for even higher resolution.

The proposed soft $x$-ray FTS system should provide the oppcrtunity to discover many new extremely narrow He resonances, now accessible only by theory, and to measure tiue line shape parameters. Of special interest will be the region of high $\mathrm{N}$ where Rydberg series converging to different and/or the same thresholds interact strongly leading to anomalies in the line width, asymmetry, cross section, energy, and quantum defect. Similar autoionizing resonances with intrinsically more complicated structure should be present in other atoms. Soft x-ray FTS could also be used to study metastable states in helium and the effects of strong external fields on the electron-elec ron correlation. In combination with molecular beam sources, soft x-ray FTS will also allow the study of specific molecular states with unprecedented resolution. 


\section{ESSENTIALS OF FOURIER TRANSFORM SPECTROSCOPY (FTS)}

The essence of a FTS system is shown in Fig. 2. An optical signal $i(t)$ (the input) impinges on the sample whose transmission spectrum is to be determined. (In our proposed experiment this incoming beam would come from the exit slit of a low-resolution monochromator.) A second signal $o(t)$ (the output), which we represent as a real optical field, emerges and is divided into two parts of nominally equal intensity, one of which is delayed by a "lag" time $\tau$. The two parts, $o(t) / \sqrt{2}$ and $o(t-\tau) / \sqrt{2}$, are then recombined (each having its intensity halved in the process) and are detected by a detector which measures the intensity $I(\tau)$ of the optical signal. Thus, neglecting losses, we have

$$
I(\tau)=\left\langle\frac{o(t)}{2}+\left.\frac{o(t-\tau)}{2}\right|^{2}\right\rangle
$$

or

$$
I(\tau)=\frac{1}{2}\left\langle\left. b(t)\right|^{2}\right\rangle+\frac{1}{2}\langle o(t) o(t-\tau)\rangle
$$

where the pointed brackets denote a time average. The measured intensity thus consists of a DC term, equal to sum of the intensities of the two beams taken separately, plus an interference term which is equal to the autocorrelation function $R_{o}(\tau)$ of $o(t)$. If $o(t)$ were a pure sine wave then $I(\tau)$ would be a pure cosine fringe pattern. If $o(t)$ were narrow band, as in our proposed experiment, then the cosine fringe pattern would have a slowly-varying envelope function modulated on to it.

We can transform the relationship (1) if we wish by the substitution $\tau=x / c, c$ being the velocity of light. We denote the spatial frequency corresponding to $x$ by $\sigma$ which is expressed in cycles per unit length (wavenumbers).

Since $i(t)$ is assumed to represent a stable light beam, it is a sample function of a stationary random process whose power spectrum we denote by $B_{i}(\sigma)$. Similarly, $o(t)$ is a filtered form of the process with power spectrum $B_{o}(\sigma)$. We therefore have according to the fundamental theorem of filtered random processes

$$
B_{o}(\sigma)=B_{i}(\sigma)|H(\sigma)|^{2}
$$

where $B_{i}(\sigma)$ is expected to be known and $H(\sigma)$ is the transfer function of the sample which is regarded as a linear filter. Substituting for the autocorrelation function in (1) using the Weiner-Kintchin theorem and using the fact that both $R_{o}(\tau)$ and $B_{o}(\sigma)$ must be real and even functions (because $o(t)$ is real) we obtain the basic, albeit somewhat idealized, description of FTS

$$
I(x)=\frac{I(0)}{2}+\int_{0}^{\infty} B_{o}(\sigma) \cos (2 \pi \sigma x) d \sigma .
$$

The quantity $I(x)-I(0) / 2$ is known as the interferogram, so (2) and (3) state that the product of the input spectrum and the desired intensity absorption spectrum is related to the interferogram by a cosine transform.

Consider the application of (3) to a wave of spatial frequency $\sigma_{0}\left(=1 / \lambda_{0}\right)$ and temporal frequency $c \sigma_{0}$. Now replacing $x$ with $v t=(d x / d t) t$ in equation (3), the temporal frequency of the detector output is seen to be $v \sigma_{0}$. Thus the action of the interferometer is to transform the temporal frequency of the incoming light by a factor $v / c$. In practice $v$ can be chosen so that the detector output is in the audio range and for narrow-band input light the detected signal will have the form of a modulated carrier wave. In our experiment with incoming light of about $2 \times 10^{5} \mathrm{~cm}^{-1}(20 \mathrm{~nm}$ wavelength), we could thus obtain an output at $1 \mathrm{kHz}$ by using $\nu=20 \mu \mathrm{m} / \mathrm{sec}$. 


\section{SAMPLING AND NOISE IN THE FTS}

Much of the original motivation for building FTS instruments for use in the infra red and visible/near UV was the different way the FTS handles noise compared to sequential grating-based spectrometers [17]. In order to discuss this quantitatively, we need to recast the problem in discrete terms which means that we must sample the interferogram at sufficiently small intervals. The general effect of such sampling of a function on its Fourier transform is explained in Fig. 3. The Fourier transform of a function sampled at intervals $\tau$ is equal to the transform of the continuous function replicated at intervals $1 / \tau$. According to the sampling theorem, if the function $f$ is band-limited with bandwidth $\sigma_{\max }$ then it is necessary to sample it at a rate higher than the Nyquist frequency $\left(2 \sigma_{\max }\right)$ (i.e. $\left.1 / \tau>2 \sigma_{\max }\right)$ in order to avoid overlap of the replicas, and thus allow an exact recovery of the original function [18].

If a sampling frequency below the Nyquist frequency is chosen then spectral features will appear at frequencies different than their true ones (aliasing) due to the overlap of the replicas. However, if the spectrum is narrow band, some degree of overlapping can be tolerated where the overlapped parts of the spectrum happen to be empty (Fig. 3). It is common practice in FTS to take advantage of this and we intend to do it also in our experiment. The use of a bandwidth $\Delta \sigma$, according to Fig. 3, would require a sampling frequency of only $2 \Delta \sigma$ rather than $2 \sigma_{\max }$. For our experiment we propose to use the unusually low value of $\Delta \sigma=0.0015 \sigma_{\max }$. In order for this to work properly, two significant conditions have to be maintained. First, the same accuracy in the indexing of $x$ must be maintained as if all frequencies up to $\sigma_{\max }$ were present. Second, the nominally empty parts of the spectrum must be actually empty of both signal and noise or the noise from all of the replicas will be additive. This can be ensured by means of an antialiasing filter which we propose to apply in the $x$ domain before taking the transform. We will describe the implementation of this device in section 10 .

For our analysis of the effect of noise, we will assume for simplicity that all frequencies up to $\sigma_{\max }$ are present and that the interferogram is represented by $N$ numerical values resulting from sampling at the Nyquist frequency $2 \sigma_{\max }$. To simplify the notation, we define the record length $\left(N / 2 \sigma_{\max }\right)$ to be the unit of length. The sampling interval then becomes $1 / N$ and the sampling frequency is $2 \sigma_{\max }=N$. It follows that the sampling interval in frequency space, $2 \sigma_{\max } / N$, is equal to 1 cycle per record length and so the frequency space sampling positions are at $\sigma_{m} \equiv m$. In this notation the definition of the discrete Fourier transform [18] is

$$
F_{m}=\frac{1}{N} \sum_{n=0}^{N-1} f_{n} e^{-2 \pi i m n / N}
$$

with inverse

$$
f_{n}=\sum_{m=0}^{N-1} F_{m} e^{2 \pi i m n / N} .
$$

When $m=0$ in (4) or $n=0$ in (5) we have

$$
F_{0}=\frac{1}{N} \sum_{n=0}^{N-1} f_{n}=\bar{f} \quad \text { and } \quad f_{0}=\sum_{m=0}^{N-1} F_{m}=N \bar{F}
$$

and Rayleigh's theorem takes the form

$$
\sum_{n=0}^{N-1}\left|f_{n}\right|^{2}=N \sum_{m=0}^{N-1}\left|F_{m}\right|^{2} \text { or } f_{r m s}=\sqrt{N} F_{r m s} .
$$

For cases of interest to us (weak absorption), the detected photon rate is close to $I(0) / 2$ most of the time (see Fig. 2) so provided that shot noise is the dominant noise process, the interferogram can be regarded as the true signal combined with constant additive white gaussian noise with zero mean and 
variance $\left(\varepsilon^{2}\right)$ equal to the number of photons measured at each data point, $I(0) / 2$. So using (6) and defining $[S / N]_{i f}^{x=0}$ as the interferogram signal-to-noise ratio at $x=0$, we have

$$
[S / N]_{i f}^{x=0}=\frac{I(0)}{2 \varepsilon}=\frac{N \overline{B_{o}}}{\varepsilon} .
$$

When we take the transform to obtain the spectrum, we will get the sum of the transform of the interferogram and of the noise. The transform of zero-mean white gaussian noise of variance $\varepsilon^{2}$ has the following properties [19]: (i) its real and imaginary parts are gaussian distributed with mean zero and variance $\varepsilon^{2} / 2 N$, (ii) its magnitude is independent of frequency (the whiteness quality) and, by (7), has a mean square value of $\varepsilon^{2} / N$ and (iii) the magnitude is Rayleigh distributed with mean $\varepsilon / \sqrt{\pi / 4 N}$ and variance $(1-\pi / 4) \varepsilon^{2} / N$. Thus we can write [20-22]:

$$
[S / N]_{\text {spect }}=\frac{B_{o m}}{\varepsilon \sqrt{(1-\pi / 4) / N}}=k\left(\frac{B_{o m}}{\overline{B_{o}}}\right)\left(\frac{1}{\sqrt{N}}\right)[S / N]_{i f}^{x=0},
$$

where $k$ is a constant of order unity ( $=2.2$ for our choice of definitions) and the second factor is the socalled filling factor equal to the ratio of the local to the average value of the spectrum. For absorption spectroscopy with weak absorption, the latter would be approximately unity. In such a case, we have the important conclusion that the signal-to-noise ratio in the spectrum is about $\sqrt{N}$ times worse than in the interferogram at $x=0$. Qualitatively this arises because the noise contributions at all the sample points of the interferogram are superposed incoherently when the sum involved in taking the transform is evaluated.

We are interested in calculating the signal-to-noise ratio at an arbitrary value of $x$ for an absorption spectrum with a fractional input bandwidth $W_{i n}$ (say). We can account for $x \neq 0$ by multiplying by the modulation, $\mu=(I(x)-I(0) / 2) /(I(0) / 2)$. So setting the filling factor equal to unity, $I(0) / 2 \varepsilon$ equal to $\sqrt{\Phi / N}$ (where $\Phi$ is the total number of detected photons in the experiment) and $N=R W_{\text {in }}$ where $R$ is the intended resolving power, equation (9) becomes

$$
[S / N]_{\text {spect }}=\frac{k \mu \sqrt{\Phi}}{R W_{\text {in }}}
$$

which we will use later to assess our proposed experiment.

Equation (9) allows us to compare the signal to noise ratio in FTS with that in a sequential grating-based spectrometer. We distinguish three cases according to the exponent in the power law $\varepsilon=I^{S}$ relating the noise to the beam intensity [21].

$s=0 \quad$ noise independent of the beam intensity: detector noise

$s=0.5$

$s=1$ noise proportional to the square root of the beam intensity: shot noise

noise proportional to the beam intensity: source noise
$\sqrt{N}$ gain in signal-to-noise ratio: the multiplex or Fellgett advantage

no advantage

$\sqrt{N}$ loss in signal-to-noise ratio: the multiplex disadvantage

It is our goal that our experiment will be shot-noise limited in which case we expect no signal-to-noise advantage compared to a grating device. The table also shows that FTS is vulnerable to source noise which must therefore be kept very low. Our approach will be to choose a mirror driving velocity so that the FTS information will be transmitted at a frequency at which the source has low noise.

Another claimed advantage of FTS is that it has a much higher phase-space acceptance (étendue) than a sequential spectrometer; the "Jacquinot advantage". However, this advantage will often not be 
applicable when the comparison is with a grating instrument fed by a modern synchrotron radiation source. This is because the source emittance is usually so low that the entire photon beam can be accepted by the grating spectrometer which of course cannot be improved on. Thus our motivation for choosing FTS is its potential for higher resolution and not the Jacquinot or Fellgett advantage.

\section{OPTICAL LAYOUT:}

The optical layout of the soft $x$-ray interferometer experiment is shown in Fig. 4. The sample in the gas cell is illuminated by soft $x$-ray radiation with a bandwidth of $0.1-1 \%$ from the spherical grating monochromator on ALS bending magnet beam line 9.3.2. Two thin windows $(\approx 100 \mathrm{~nm})$ at the inlet and outlet of the gas cell (with a gas pressure of 1-100 mbar) isolate the sample from the rest of the components which are at UHV. Such a design has already been used by the authors to measure the photoabsorption spectra of helium [23]. The beam transmitted by the helium sample is the input to the interferometer which is a deformed Mach-Zehnder system with grazing reflections. The shape of the rhombus $\mathrm{ABCD}$ is determined by the reflectivity of the optical elements and its size is determined by the desired beam path difference (resolving power). The beam splitters are two transmission-grating devices (BS1 and BS2) which we plan to make from flat silicon mirrors with a series of slots as discussed separately. The optical path difference is created by moving the four mirrors (M1, M2, M3, \& M4) which are mounted rigidly together on a table, along BD in either direction relative to the rest of the components which are mounted separately. The diffracted beams from the grating beam splitters are focused at the horizontal slit (SLIT2) in front of the detector by a vertically deflecting spherical mirror. This is to reject all beams other than zeroth order, and thus eliminate ambiguities in the path difference.

\section{MIRROR REFLECTING EFFICIENCY}

Molybdenum is about twice as good a reflector as gold in the 10 to $20 \mathrm{~nm}$ range and has high reflectance down to $10 \mathrm{~nm}$ for a grazing angle of $20^{\circ}$ (see Fig. 5). Using $20^{\circ}$ at the beam splitters implies $10^{\circ}$ at the mirrors which provides a total refiection efficiency of about 0.4 at $20 \mathrm{~nm}$ with clean optics. It is interesting to note that the reflection efficiency of carbon is rather good around $20 \mathrm{~nm}$ and so the loss of flux due to carbon contamination of the mirrors would not be all that serious. Furthermore, it is possible to use less reactive coatings such as $\mathrm{Mo}_{2} \mathrm{~B}, \mathrm{MoB}, \mathrm{Mo}_{2} \mathrm{C}$ or $\mathrm{MoC}$ with reflection efficiencies almost identical to clean Mo.

\section{BEAM SPLITTERS}

Our proposal for a beam splitter for the FTS system is based on wavefront division using reflection by the bars and transmission through the slots of a transmission grating structure. Our plan for fabricating such a device is to apply microfabrication techniques to a specially shaped silicon single crystal. As shown in Fig. 6, each beam splitter consists of a rigid flat mirror (slope error $<0.75 \mu$ radian) with a series of slots of width $25 \mu \mathrm{m}$ etched in part of the area. The etching technique proposed for making the slots is similar io LIGA except that we plan to utilize preferential etching of the silicon (110) surface by an alkali solvent which etches along all crystallographic directions except (111). This technique has been previously utilized successfully [24] with aspect ratios similar to the value of around 20 that we require. We plan to start with a polished mirror of $0.5 \mathrm{~mm}$ thickness in the active part and 
much thicker everywhere else, as shown in Fig. 6 and then apply the etching technique in the thin region to obtain the desired transmission grating structure.

\section{TOLERANCES}

Following Chamberlain [25] we have analyzed the effect of wave front errors in terms of a spectral distortion factor $D(\sigma)$ which multiplies $B_{o}$ in (3). In order to establish tolerances, we must set limits to the value of $D(\sigma)$ and we choose

$$
D(\sigma)>0.9 \text { for } \sigma=10^{6} \mathrm{~cm}^{-1} \text { (same as } \lambda=10 \mathrm{~nm} \text { ) and } r=1 \mathrm{~mm}
$$

where $r$ is the radius of the system aperture.

Like most spectroscopic systems, the interferometer is fairly immune to translational errors but very sensitive to rotational ones. The basic type of error is a tilt by an angle $e$ of one of the interfering wave fronts with respect to the other one. Applying (11) gives

$$
e<\frac{0.911}{2 \pi \sigma r}
$$

which leads to $e<1.5 \mu$ radians in our case. Roughly, a relative tilt of a wave front should produce less than a quarter of a wave of path error at the edge of the aperture. Thus $e r<\lambda / 4$ or $e<2.5 \mu$ radians which is in reasonable agreement.

Based on this and other arguments, we estimate the values in the table below. The allowed beam angle comes from an analysis, again by Chamberlain, of the change of path difference with angle. We consider table roll to have a negligible effect because the initial displacement of each mirror is in its own plane and table yaw to be relatively harmless because, for each beam, the two mirrors produce equal and opposite effects in the manner of a pentaprism. The most challenging geometrical tolerances appear to be the beam splitter surface and the pitch error of the moving table.

Incoming beam:

Angle

Width

Relative tilt of interfering wave fronts

Mirror surfaces:

Slope

Beam splitter surfaces:

Slope

Moving table:

Pitch

Roll

Yaw

$$
\begin{aligned}
& \pm 1.4 \text { milliradian } \\
& 2 \mathrm{~mm} \text { (fuil width) } \\
& 1.5 \mu \text { radian }
\end{aligned}
$$

$\pm 0.5 \mu$ radian (1.0 peak to valley)

$\pm 0.75 \mu$ radian (1.5 peak to valley)

$\pm 1.0 \mu$ radian

large

large

\section{MECHANICAL MOTION DEVICE}

The desired \pm 1 -cm path difference requires a linear motion of $1.5 \mathrm{~cm}$ with a pitch error below 1 $\mu$ radian. This is intermediate in scale between the requirements of FTS in the near UV (40 cm and 3 $\mu$ radian) [3] and of interferometry in the hard x-ray region $(0.01 \mathrm{~cm}$ and $0.001 \mu$ radian) [26] both of which have been successful. We propose to use a monolithic flexural mechanism similar to ones used in hard $x$-ray interferometry. However, although we would prefer to use flexural hinges of the doublecircle type, we are obliged by stress considerations, resulting from our larger amount of motion, to 
flatten the hinges by inserting the straight section in the center as shown in Fig. 7. Earlier mechanisms of this general shape have used both flexible-strip [27] and double-circle [28] type hinges. For the latter case, the pitch error per unit of motion was found to be $0.1 \mu \mathrm{radian} / \mathrm{mm}$ initially but this was reduced to $0.005 \mu \mathrm{radian} / \mathrm{mm}$ after optimizing the point of application of the driving force.

The size of the mechanism is determined by the geometry of achieving a \pm 1 ini path difference and will be about $\mathrm{J.4} \mathrm{m}$ in the longest direction. Given this we can consider what materials are aviilable in suitable size. We have found four candidate classes of material exemplified by the following: tool steel type S7, alloy steel 4340, maraging steel C-300 and aluminum alloy 7075-T651. All are rather high-stress materials and although the aluminum has a 3-4 times lower stress rating it generates correspondingly less stress for a given strain due its smaller elastic modulus.

An important consideration for this application is fatigue. We have estimated the strain range corresponding to $10^{5}$ cycles to failure for the above four materials by means of the method of universal slopes [29] with the following results: tool steel $\pm 0.42 \%$, alloy steel $\pm 0.47 \%$, maraging steel $\pm 0.50 \%$, aluminum alloy $\pm 0.34 \%$. The conclusion is that all the above four materiais are roughly equally suitable from a fatigue point of view.

For high repeatability of motion, one would like to have a material with minimal nonelastic behaviors such as room temperature creep, internal damping etc. However, we have had difficulty finding quantitative information on these aspects.

Another important question is the type of driver. Our intention is to drive the system continuously. The uniformity of the velocity does not directly determine the position measurement but it does have second order effects which mandate a reasonably high uniformity. The primary requirements are smoothness and freedom from periodic errors [3]. Our present thinking is to use a hydraulic drive but this will require some design study to make it compatible with the ultrahigh vacuum environment.

For indexing the motion we propose to use heterodyne laser interferometry. The resolution of a few tenths of a nm provided by commercially available systems appears to be sufficient for our purposes.

\section{SIGNAL ACQUISITION}

The signal processing requirements which have emerged so far may be summarized as follows: a scan of $\pm 1 \mathrm{~cm}$ of path difference with incoming light at approximately 20nm will generate a signal consisting of $10^{6}$ waves at $1 \mathrm{kHz}$ from the detector and will take 1000 seconds. The spectral information will be in the form of a slowly varying envelope function with bandwidth $(\Delta \sigma)$ equal to $0.15 \%$ of the carrier frequency or $1.5 \mathrm{~Hz}$. The sampling interval required by the modified sampling theorem described earlier is $1 /(2 \Delta \sigma)$ which is $0.33 \mathrm{sec}$ or 330 periods of the carrier wave. Our strategy will be to massively oversample the detector signal and then reduce the data digitally to a much smaller data set representing the interferogram sampled at close to the sampling-theorem rate.

The signal processing scheme is shown in Fig. 8. The current from the detector will be amplified using a low-noise current amplifier coupled to an analog-to-digital converter (ADC). The ADC will be a 16-bit integrating delta-sigma type running at full sampling rate to capture essentially all of the detector signal. A sampling rate of $100 \mathrm{kHz}$, a modest number for modern ADC's, would be integrating a signal over $1 / 100$ of a period of the carrier wave. The ADC would be preceded by a low-pass antialiasing filter to remove any signal of frequency greater than $100 \mathrm{kHz}$ resulting from noise in the earlier stages. The digital signals produced by this procedure, indexed by the laser interferometer, will be filtered by a digital signal processor chip in real time and stored in a computer for display and further analysis. This filter is a second antialiasing filter whose action was explained in section 4 and which is to be applied to the measured interferogram in the time domain (which is equivalent to the $x$ domain). Its purpose will 
be to remove signals which lie outside the band $\Delta \sigma$. The design of such filters has been discussed for example by Brault [21]. An advantage of the great length of the fundamental sampling interval is that one will be averaging over many indexing measurements by the laser interferometer so that random errors in that process can be reduced.

\section{DETECTOR}

To detect the interferogram, the number of photons over the above-mentioned 10-microsecond time window has to be determined. In order to achieve the lowest noise possible, we propose to use an ion chamber or a semiconductor photodiode. In the first case, the photon flux will be measured as the total photoionization current using a two-plate ionization chamber of several $\mathrm{cm}$ active length filled with $\mathrm{He}$ gas. One electrode will be held at a potential of about $+20 \mathrm{~V}$, while at the other, the photoionization current will be measured by a current amplifier. The silicon n-on-p photodiode is easier to use and has a high quantum efficiency in the soft $x$-ray range (17 electron-hole pairs per photon at $65 \mathrm{eV}$ ). It also is characterized by a low noise-equivalent-power, typically $7 \times 10^{-14} \mathrm{~W} / \sqrt{\mathrm{Hz}}$ at $10 \mathrm{eV}$.

The choice between photodiode and ion chamber is based partly on the fact that they measure different things. The ion chamber measures the absorbed signal while the silicon photodiode would measure the transmitted signal. The filling factor term in equation (9) is about unity for a transmission measurement but much larger (better) for an absorption signal measurement. This is a great advantage for the ionization chamber but we are still studying its practicality in other respects such as speed.

A second aspect of the comparison between the two detection schemes is the degree to which they can approach the shot-noise limit. The performance of a propane filled proportional counter and a room-temperature silicon photodiode was tested with a laser-produced plasma as a source of $100 \mathrm{eV}$ photons [30]. The measurements showed that both detectors are shot-noise limited at $10^{4}$ photons per second. At a lower photon rate, detector noise in the silicon photodiode sauses an increase above the shot-noise-limited dark current. The proportional counter is shot-noise-limited even at very small photon rates. Preliminary analysis of helium absorption data taken by the authors with both types of detector indicate that the variance of the data taken with an ionization cell is closer to the shot noise limit than with the photcdiode. Before a conclusion on which detector to use can be reached, further analysis of all participating noise sources such as the fluctuations of the synchrotron beam and electronic noise from the ampiifier has to be done.

\section{PROJECTED PERFORMANCE AT AN ALS BENDING MAGNET BEAM LINE}

In order to estimate the fractional modulation in our interferogram, we performed computer simulations of an absorption spectrum using the $2(0,1)^{+}$resonances in He. The position and shape of these resonance lines were extrapolated based on the parameters given in [13]. By applying the Rydberg formula, we estimated that with a resolving power of $E / \Delta E=5 \times 10^{5}$ resonances with up to $n=57$ could be observed. The input spectrum for the FTS was calculated for a band width covering resonances with $6 \leq$ $n \leq 60$ which absorb $10 \%$ of the incoming photons at their maximum. The Fourier transformation of the transmission spectrum showed a fractional modulation of about 0.005 .

We are now in a position to estimate the signal to noise ratio of our experiment using equation (10). Taking the realistic values $\Phi=5 \times 10^{14}$ photons per 1000 seconds per $0.15 \%$ bandwidth for our bending magnet beam line, $\mu=0.005, k=2.2$ and $R=5 \times 10^{5}$, we obtain the very promising value of 330 for the signal to noise ratio. The key assumptions involved in this are that the interferometer approximates its ideal geometry well enough not to degrade the value of $\mu$ and that the detection system is shot-noise limited. 
We conclude from this that our experiment will be successful if we are able to build and operate the system according to the tolerances worked out in earlier sections.

\section{ACKNOWLEDGMENTS}

The authors are grateful for the help, advice and encouragement of J. Brault, R. Deslattes, E. Kessler, R. Learner, R. P. Madden and A. Thorne. We also thank G. Kaindl for his permission to reproduce Fig. 1. This work was supported by the Director, Office of Energy Research, Office of Basic Energy Sciences, Material Sciences Division of the U. S. Department of Energy, under contract DEAC03-76SF00098. One of us (TR) thanks the Alexander von Humboldt Foundation for support through a Feodor Lynen Fellowship and Penn State University for additional support.

\section{REFERENCES}

1. A. Sommerfeld, Optics, Lectures on Theoretical Physics (Academic, New York, 1949), vol. IV.

2. K. D. Moller, T. Sears, H. T. Lui, C. J. Hirschmugl, G. P. Williams, Nucl. Instrum. Meth. A319, 384 (1992).

3. A. P. Thorne, C. J. Harris, I. Wynne-Jones, R. C. M. Learner, G. Cox, J. Phys. E 20,54 (1987).

4. U. Bonse, Generalized Michelson Interferometry, ALS Technical Note LSBVL-7, Lawrence Berkeley Laboratory (1987).

5. M. Born, E. Wolf, Principles of Optics, (Pergamon, Oxford, 1980).

6. R. P. Madden, K. Codling, Phys. Rev. Lett. 10, 516 (1963).

7. J. W. Cooper, U. Fano, F. Prats, Phys. Rev. Lett. 10, 518 (1963).

8. U. Fano, Rep. Prog. Phys. 46, 97 (1983).

9. D. R. Herrick, Adv. Chem. Phys. 52, 1 (1983).

10. C. D. Lin, Phys. Rev. A 29, 1019 (1984).

11. J. M. Rost, J. S. Briggs, J. Phys. B 24, 4293 (1991).

12. K. Richter, J. S. Briggs, D. Wintgen, E. A. Solov'ev, J. Phys. B 25, 3929 (1992).

13. M. Domke, C. Xue, A. Puschman, T. Mandel, E. Hudson, D. A. Shirley, G. Kaindl, C. H. Greene, H. R. Sadeghpour, Phys. Rev. Lett. 66, 1306 (1991).

14. M. Domke, G. Remmers, G. Kaindl, Phys. Rev. Lett. 69, 1171 (1992).

15. L. Wu, J. Xi, J. Phys. B 23, 727 (1990).

16. A. Marcias, T. Martin, A. Riera, M. Yunez, Phys. Rev. A 36, 4187 (1987).

17. J. Connes, Revue Opt. Theor. Instrum. 40, 45 (1961).

18. R. N. Bracewell, The Fourier Transform and its Applications, (McGraw-Hill, New York, 1978).

19. S. Goldman, Information Theory, (Prentice-Hall, New York, 1953).

20. J. W. Fleming, Infrared Physics 17, 263 (1977).

21. J. W. Brault, in High Resolution in Astronomy A. Benz, M. Huber, M. Mayer, Eds. (Genevia Observatory, Sauverny, Switzerland, 1985) pp. 1.

22. A. Thorne, J. Anal. Atom. Spect. 2, 227 (1987).

23. T. Reich, Z. Hussain, E. Moler, M. Biackwell, G. Kaindl, D. A. Shirley, M. R. Howells, Rev. Sci. Instrum. 64, 2552 (1993).

24. D. B. Tuckerman, PhD Thesis, LLNL report UCRL-53515, Stanford (1984).

25. J. Chamberlain, The Principles of Interferometric Spectroscopy. (John Wiley and Sons, Chichester, 1979).

26. R. D. Deslattes, in Precision Measurement and Fundamental Constants II, B. N. Taylor, W. D. Phillips, Eds. (National Bureau of Standards, Gaithersburg, 1984), Spec. Publ. 617, pp. 303. 
27. U. Bonse, E. t. Kaat, P. Spieker, in Precision Measurement and Fundamental Constants D. N. Langenburg, B. N. Taylor, Eds. (National Bureau of Standards, Gaithersburg, 1971), Spec. Publ. 343, pp. 291.

28. C. Hoffrogge, H.-J. Rademacher, PTB Mitteilungen 83, 79 (1973).

29. S. S. Manson, Experimental Mechanics 5, 193 (1965).

30. E. M. Gullikson, J. H. Underwood, P. C. Batson, V. Nikitin, J. X-Ray Sci. Technol. 3, 283 (1992).

\section{FIGURE CAPTIONS}

1. Autoionizing resonances of doubly excited He below the $\mathrm{N}=2$ threshold (IP2) of $\mathrm{He}^{+}$taken from reference [14].

2. a) Essentials of Fourier Transform Spectrometer system. b) Example of an interferogram where the output intensity is plotted as a function of path length differences.

3. The spectrum of a continous function is shown at the top left. The spectra from the same function after it has been discretely sampled are shown on the right and consist of periodic replications of the original spectrum with a degree of overlap of the replicas that depends on the sampling rate. Aliasing which normally results from sampling below the Nyquist frequency can be avoided if the original spectrum is suitably narrow-band, as shown at the bottom.

4. Schematic diagram of the soft $x$-ray interferometer experiment. S1:- beamline monochromator exit slit; BS1 and BS2:- beamsplitters; M1, M2, M3, M4:- flat mirrors; M5:- spherical focussing mirror, S2:- slit for rejecting orders other than zero diffracted by the beam splitters. ABCD is a rhombus of side $15 \mathrm{~cm}$.

5. Reflectivity of molybdenum as a function of photon energy for several grazing angles.

6. Proposed design of the transmission-grating beam splitter made from a silicon single crystal.

7. Schematic of a monolithic flexure design to produce linear motion for the FTS system. Black represents solid metal. The four flat mirrors are mounted on the central rectangle which moves relative to the outer structure where the beam splitters are mounted.

8. Schematic of the proposed data acquisition system. 


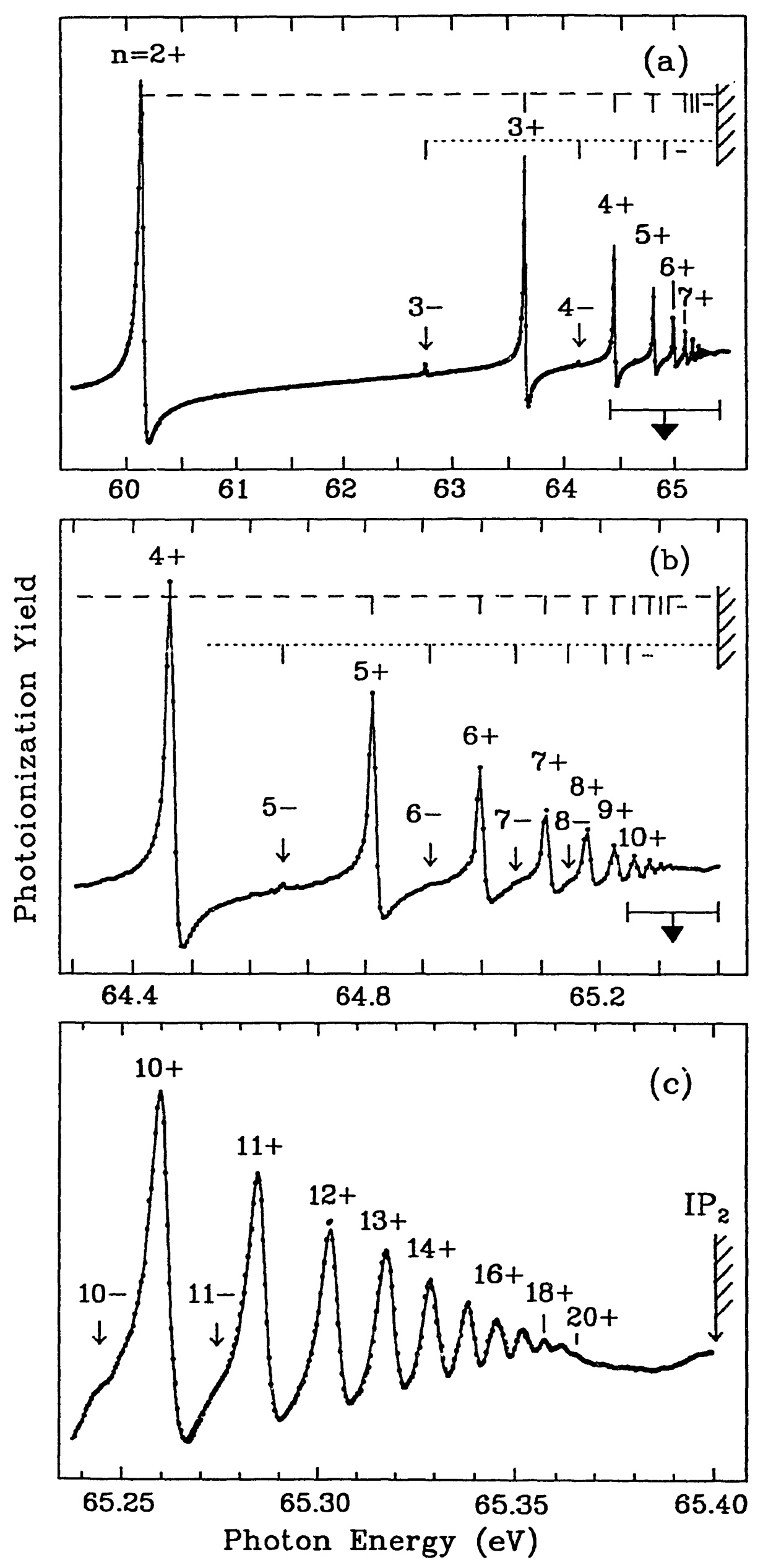

Fig. 1 


\section{(a)}

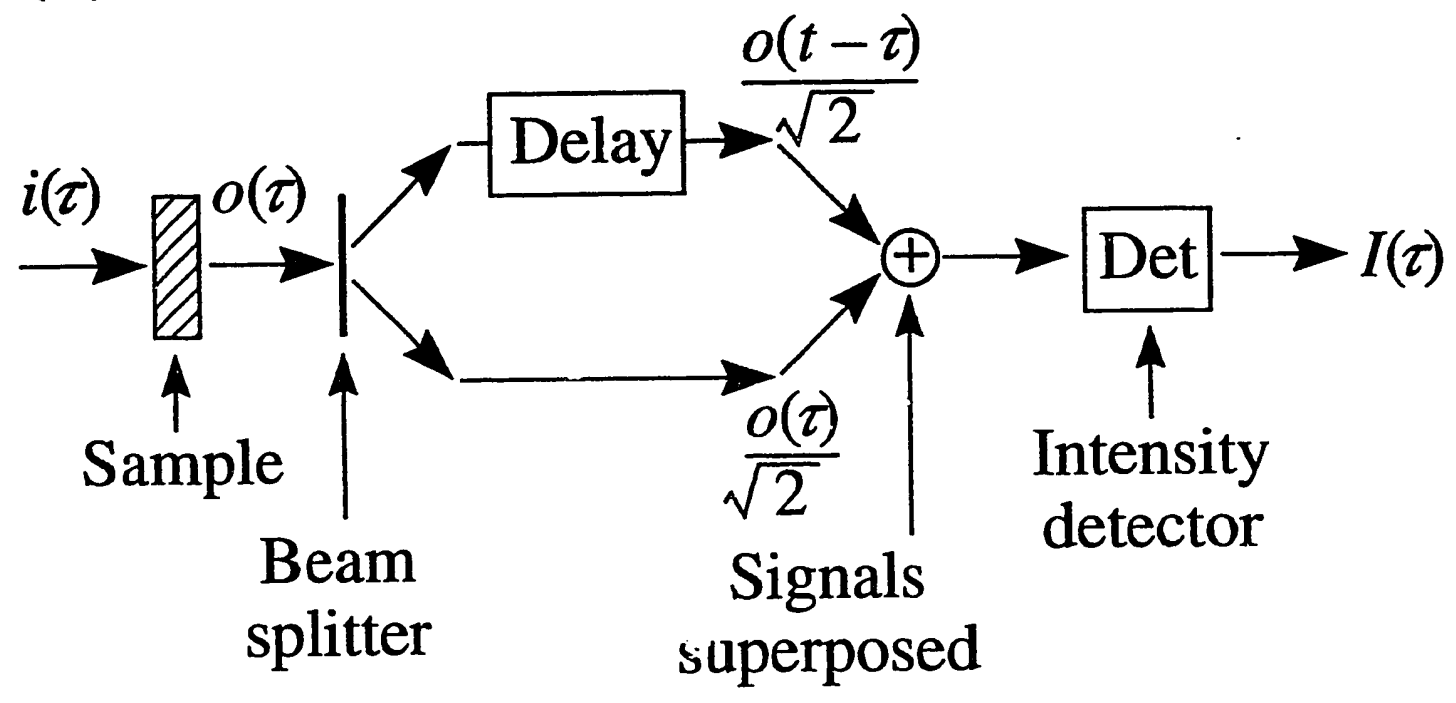

(b)

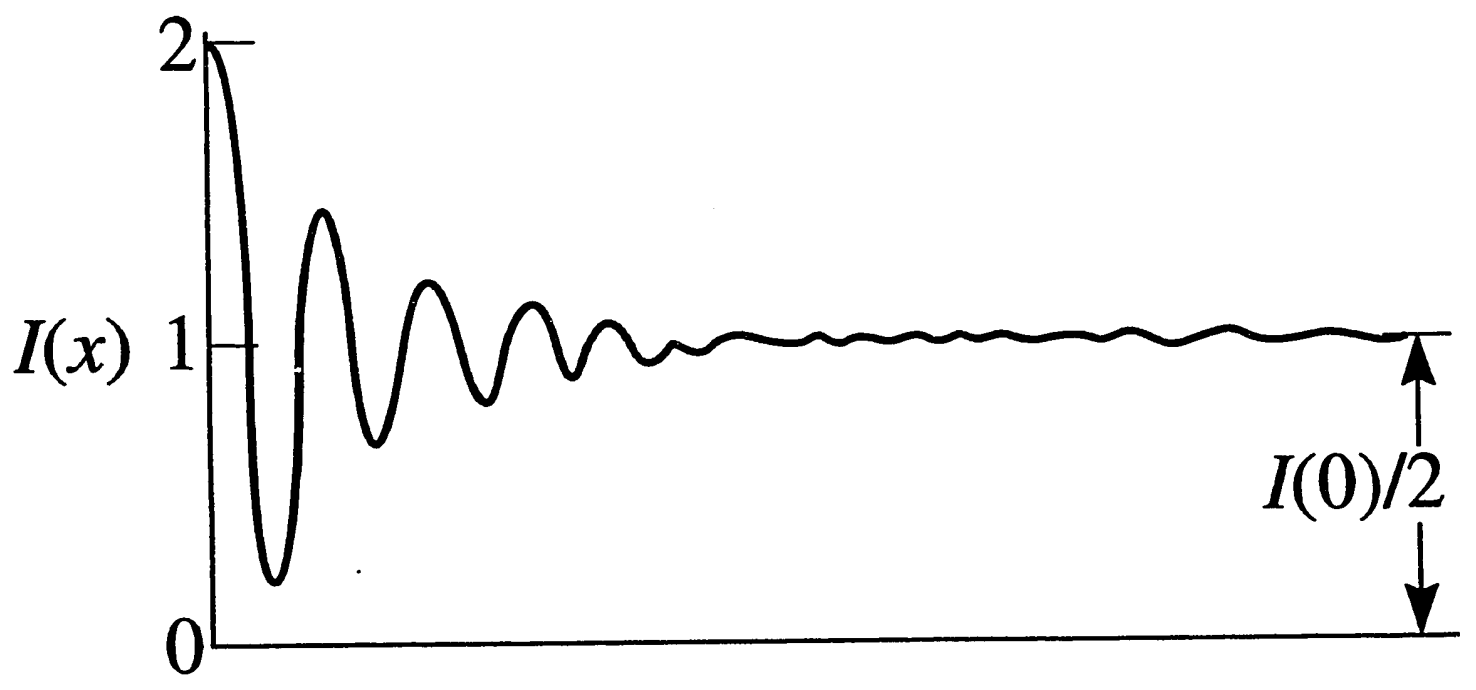

XBL 9310-4113 
Spectrum of the continuous function

$\mathscr{F}[f(x)]=F(\sigma)$

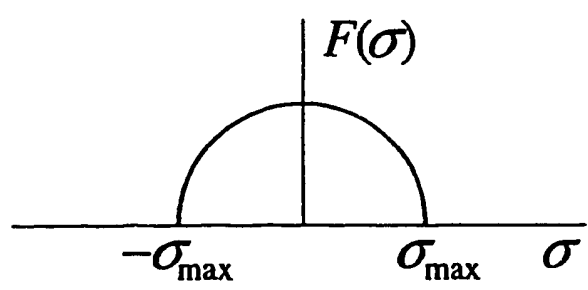

Restricted band of $F(\sigma)$

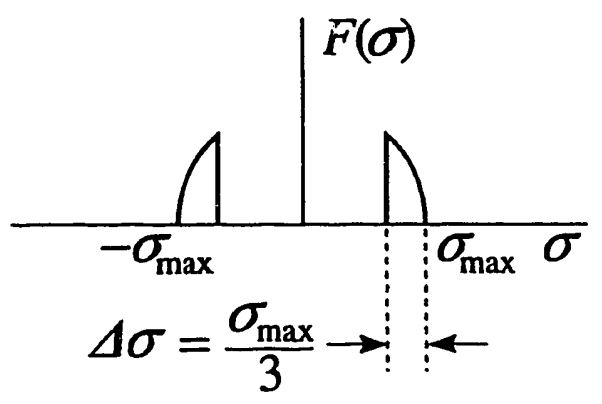

Spectrum of the sampled function

$\mathscr{F} f(x) \mathrm{III}(\mathrm{x} / \tau]=F(\sigma) *|\tau| \mathrm{III}(\sigma \tau)$

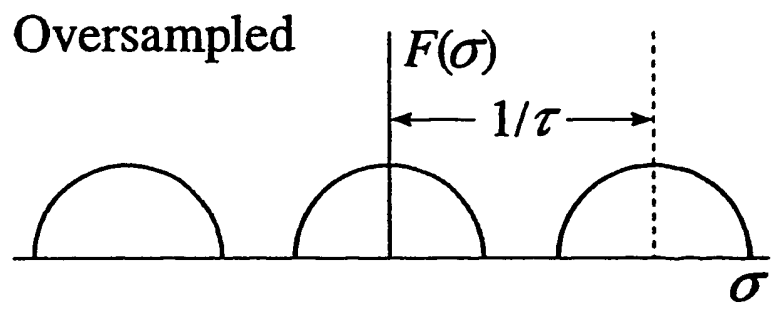

Sampled at the

Nyquist freq. $\left(1 / \tau=2 \sigma_{\max }\right)$
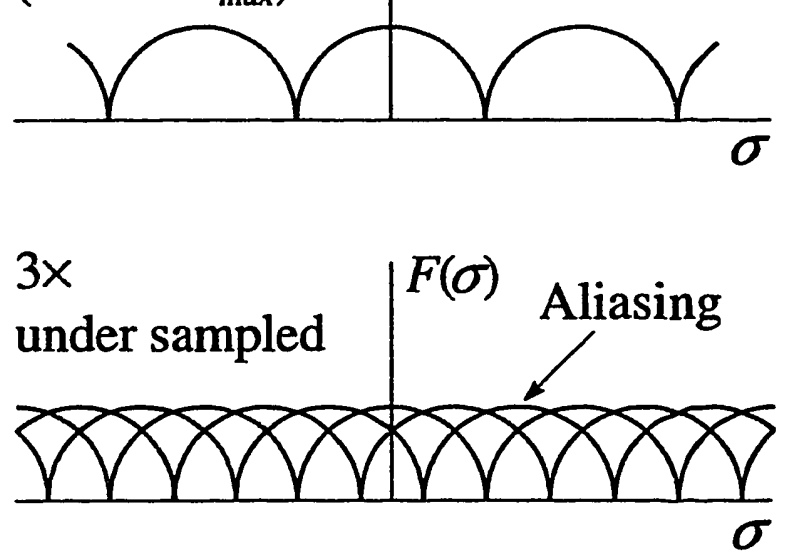

$3 x$

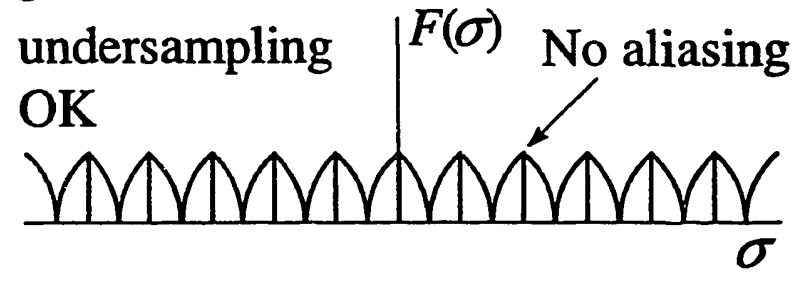

XBL $9310-4112$ 


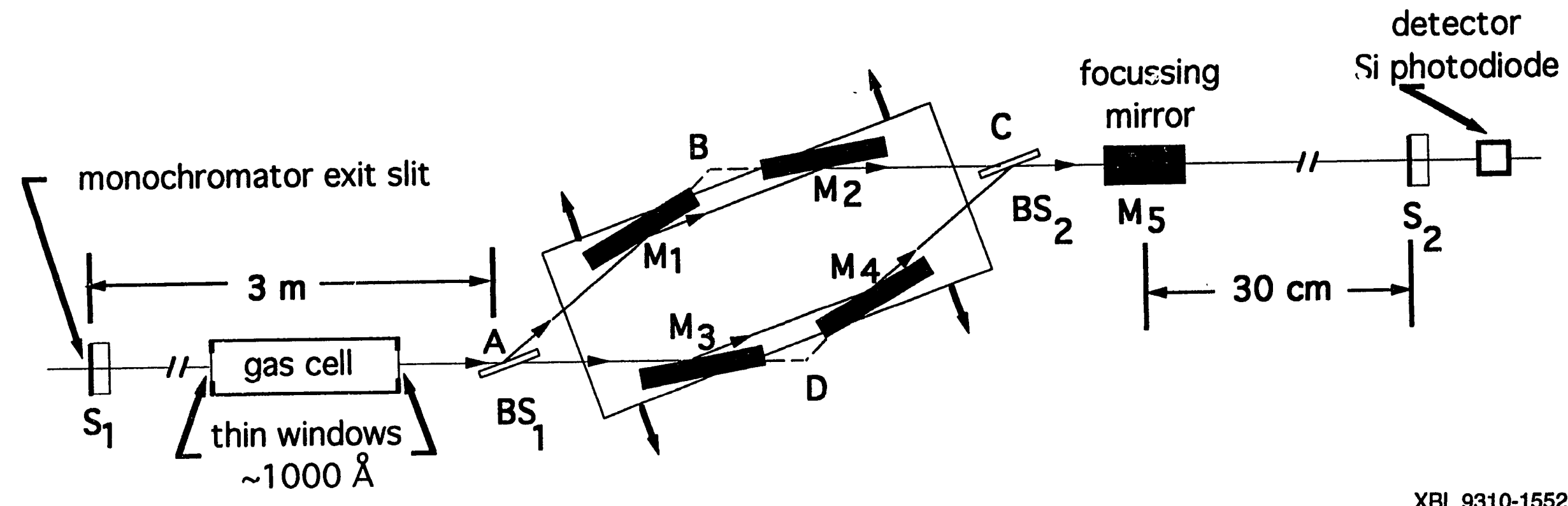

XBL 9310-1552 


\section{Reflection Efficiency}

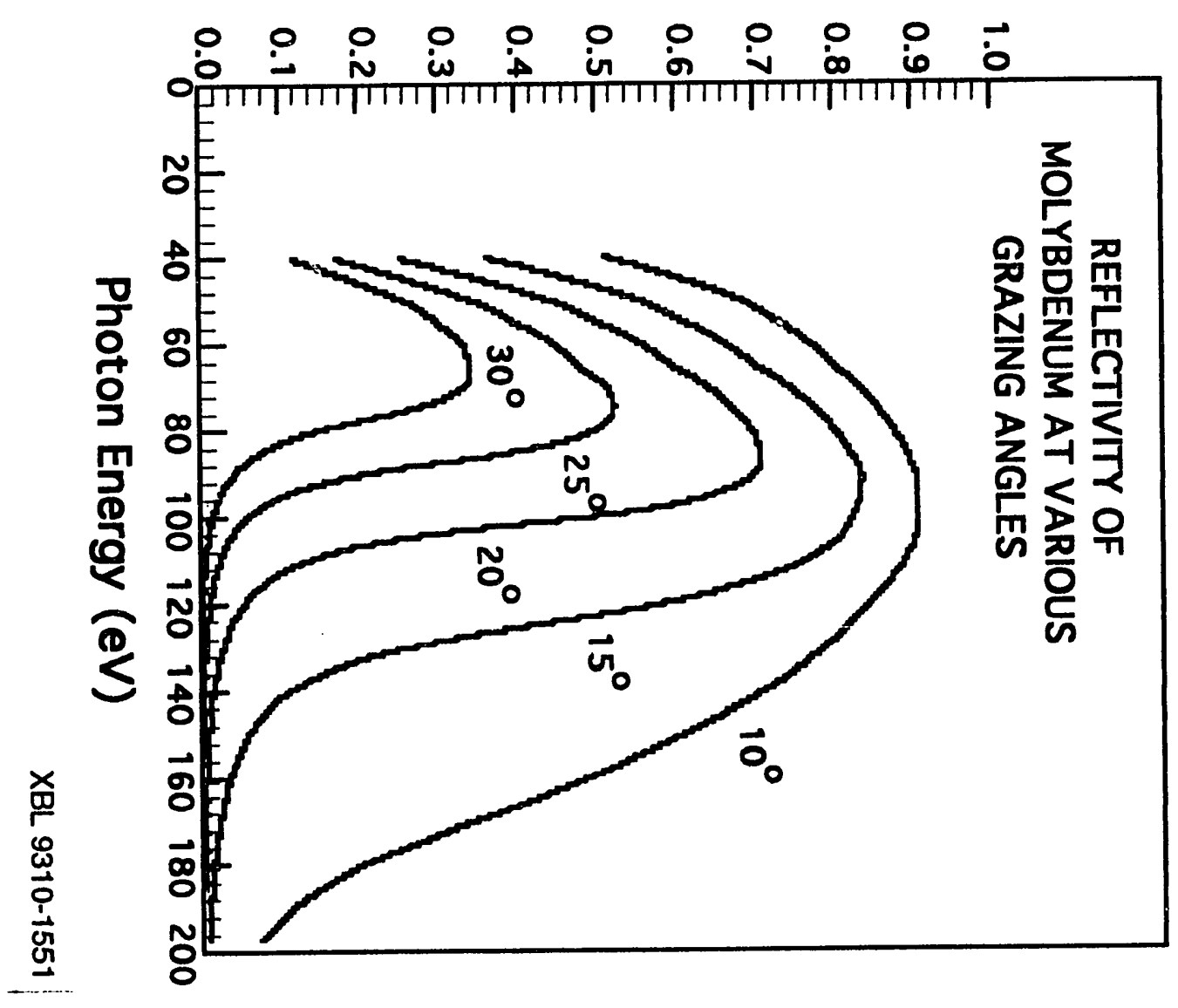



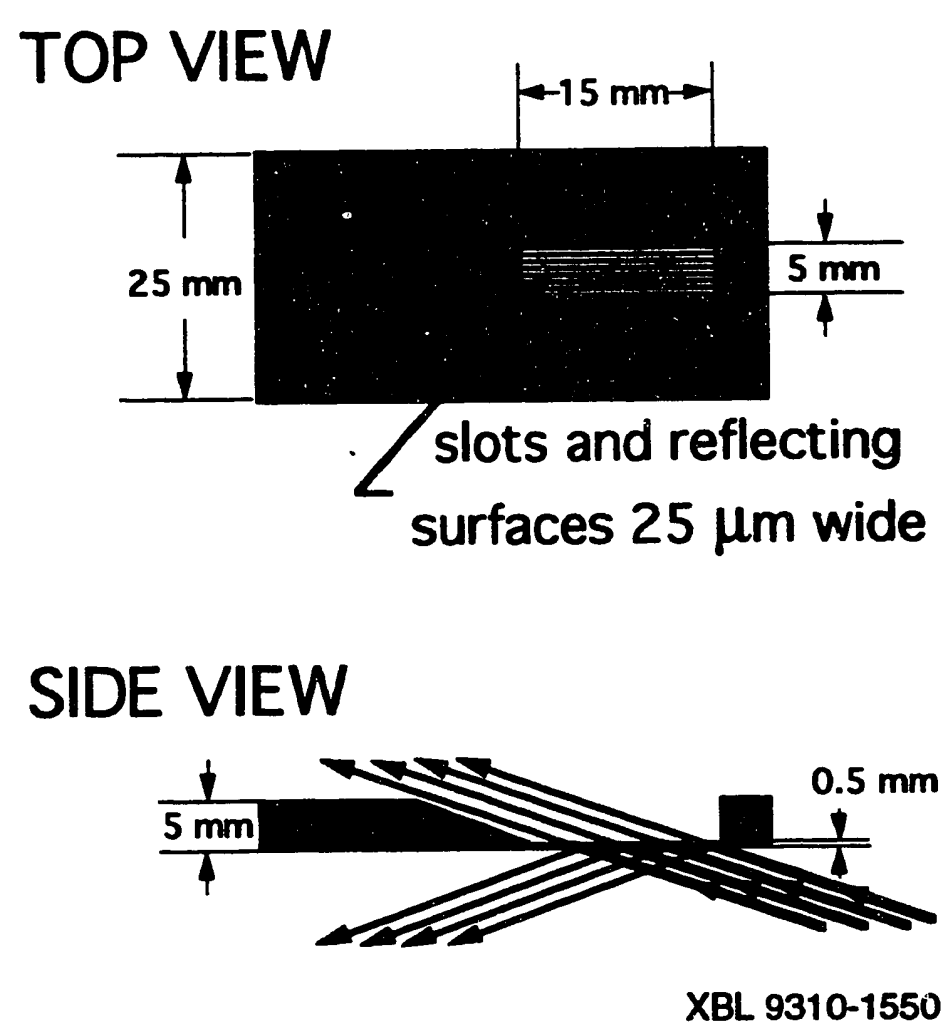

柔 


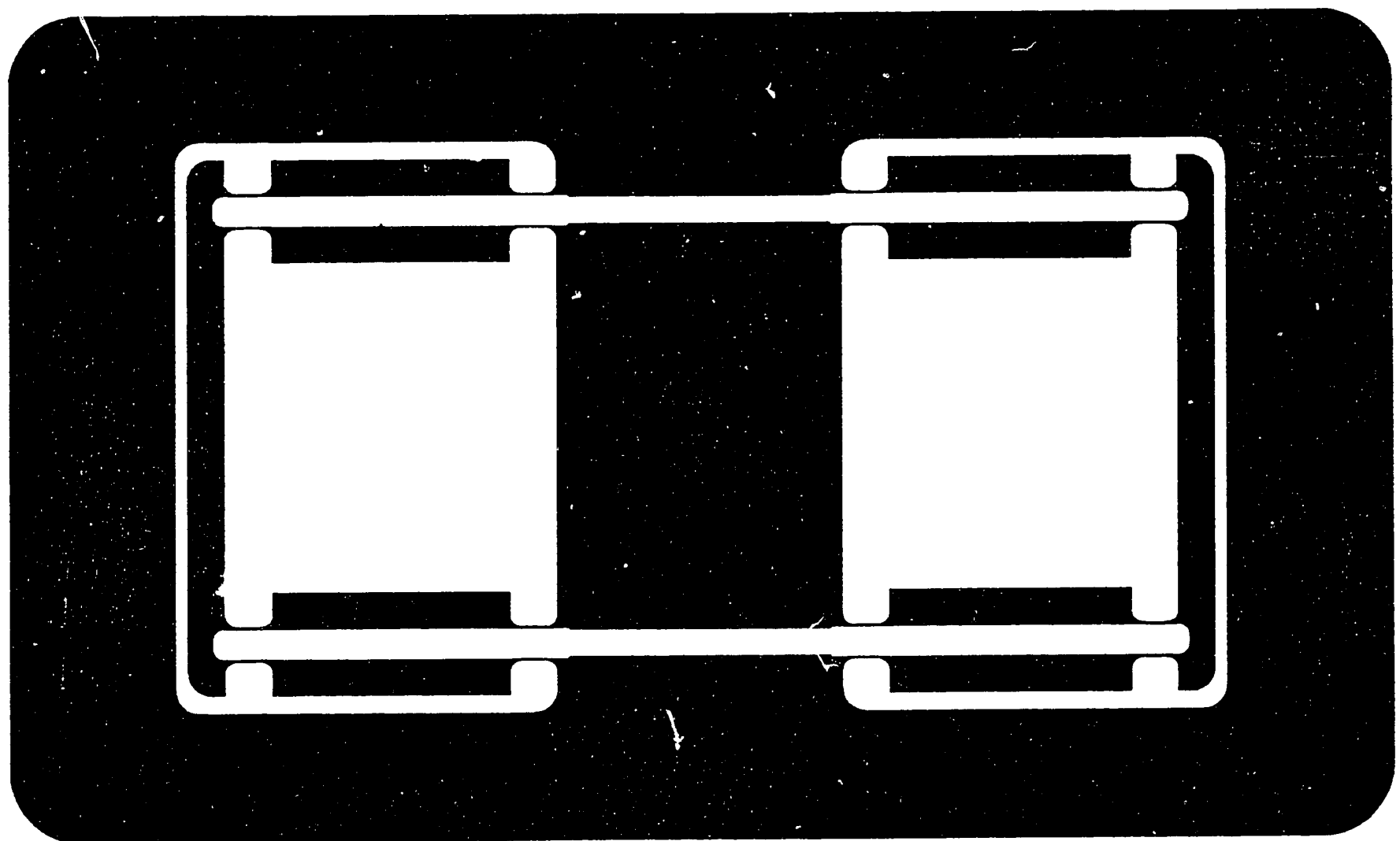

XBL 9310-1549

Fig. 7 


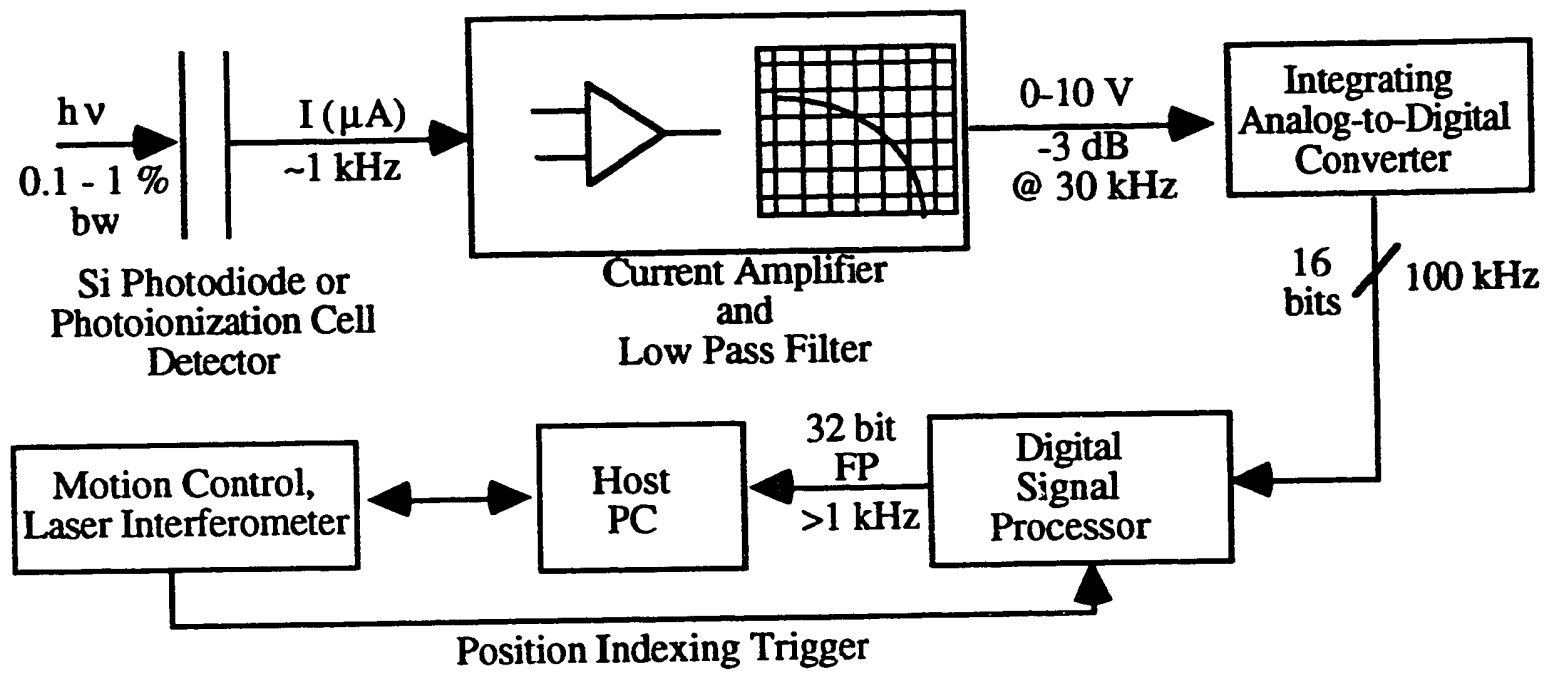

XBL 9310-1548

Fig. 8 

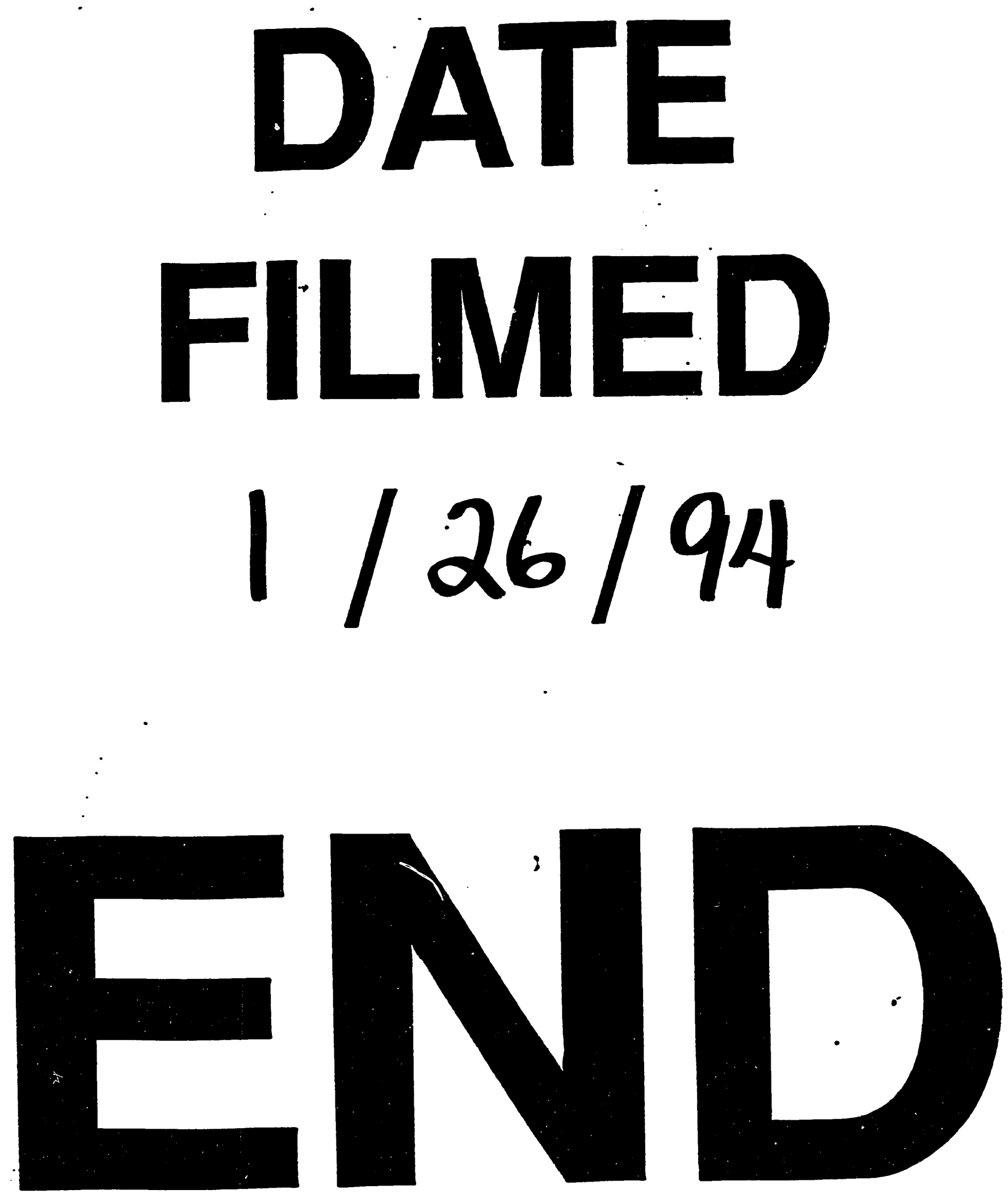